\title{
Prostate cancer: some clinical and scientific dilemmas.
}

Paul D. Abel

Imperial College London

El-Nasir Lalani

Aga Khan University, elnasir.lalani@aku.edu

Follow this and additional works at: https://ecommons.aku.edu/pakistan_fhs_mc_pathol_microbiol

Part of the Cells Commons, and the Oncology Commons

\section{Recommended Citation}

Abel, P. D., Lalani, E. (2003). Prostate cancer: some clinical and scientific dilemmas.. Prostate Cancer, 3-19.

Available at: https://ecommons.aku.edu/pakistan_fhs_mc_pathol_microbiol/431 


\title{
1
}

\section{Prostate Cancer: Some Clinical and Scientific Dilemmas}

\author{
Paul D. Abel ${ }^{*}$ and El-Nasir Lalani ${ }^{\dagger}$ \\ *Department of Surgical Oncology and Technology \\ Division of Surgery, Anaesthetics and Intensive Care \\ Imperial College, Faculty of Medicine \\ Hammersmith Campus, Du Cane Road, London W12 ONN, UK \\ Tel: 44-20-8383-2268; p.abel@imperial.ac.uk \\ ${ }^{\dagger}$ Department of Histopathology \\ Division of Investigative Sciences, Imperial College \\ Imperial College, Faculty of Medicine \\ Hammersmith Campus, Du Cane Road, London W12 ONN, UK \\ Tel: 44-20-838-2430; e.lalani@imperial.ac.uk
}

\section{BACKGROUND}

In 1994, we attended a superb meeting that brought together many researchers studying prostate cancer $(\mathrm{PCa})$. The standard of talks was outstanding. Initially, we felt elated and inspired by the data and discussions generated. Future possibilities seemed endless. On reflection however, it became obvious that almost none of the data presented, which had cost millions of pounds and taken thousands of man hours to complete, had had any immediate, medium or even long term impact whatsoever on the clinical management of patients with PCa. It was not immediately apparent why this should be. However, most of the researchers were basic science orientated and the relatively minor input of clinicians may have been an important factor. Also, many of the speakers had been previously working on molecular aspects of other tissue and organ systems, including cancers, but were exploring the possibility of applying 
their technology to prostate disease as increased PCa funding was starting to trickle through. We were also reminded of the difficulties that we had personally experienced when trying to engineer collaborations in the past. It became apparent that very clear agreements needed to be made before these could take place and the kind of trust required to develop them took some time to build. Issues such as order of authorship, principal investigator status, contributions to concepts, which group did which elements of the work and the specifics of a time frame were essential to agree before starting work. How these were resolved in practice could make a real difference to whether collaborations were short term or continued over many years and with increasing success.

So it became an aspiration to work toward developing interspecialty collaboration and help bridge the gap between clinicians and scientists. One potentially useful route would be to attempt a text, which mixed clinical and scientific contributions. Its primary objective would be to provide a clear explanation of PCa as perceived by specialists so that non-specialists could understand issues outside their areas of expertise. We felt this was more of a priority than being right up to date. Without interdisciplinary cross talk, scientists may take the path of pure molecular and cellular research, in itself a noble object but perhaps less likely to meaningfully impact upon clinical practice. At the same time, clinicians need an understanding of what science can offer and how they too have an important role to play in the process, maybe by coaxing scientific colleagues toward answering questions that could have translational significance. By working together, the sum of both groups should be greater than that achieved by either alone.

We discuss below a number of the issues we perceive that may be impeding progress.

\section{TRANSLATIONAL RESEARCH}

In the past 100 years or so there has been little in the way of meaningful advances in the clinical management of $\mathrm{PCa}$ apart from 
Huggins discovery of the androgen regulation of the prostate in the 1940 's. Yet during this period, and especially the past 25 years, there has been an explosion in biomedical knowledge. The last 10 years has brought high throughput technologies including genomics, proteomics and micro-array technologies and the entire human genome is now unravelled. But there remains a very wide gap between laboratory findings and clinical applications at the bedside and there are few parallels between major scientific advances and advances in patient management. Waldmann has said "there is a need to translate these fundamental scientific insights into new approaches for prevention, diagnosis and treatment of human disease for the benefit of patients and this can only be addressed by patient orientated clinical research". ${ }^{1}$ Why are there such problems?

\section{IMPEDIMENTS TO PROGRESS OF TRANSLATION RESEARCH}

Translation involves the application of basic science discoveries into clinically germane findings and simultaneously the generation of scientific questions based on clinical observations. ${ }^{2,3}$ Pober et al. ${ }^{2}$ believe that translation is not a straightforward process but a new form of research in its own right and therefore problems arise as a direct result. Many impediments exist and compromise progress as a consequence and some are discussed below.

There is a shortage of suitable trained individuals to undertake translational research. The skills to address this are usually not available in a single laboratory or clinical setting and Pober et al. suggest the need for academic medical centres as a model to conduct translational research. These consist of combined Medical Schools and Hospitals because such institutions tend to accommodate both clinical and laboratory based investigators. However, they are 30-40\% more expensive than non-academic hospitals. ${ }^{4}$ This is hardly surprising when the duration of training these investigators can exceed well beyond 10 years. Yet such individuals have 
increasing pressure on them to meet service rather than research needs. Focusing on science may be a major disincentive as it limits personal income and/or the ability to sustain critical skills, for example in operative surgery, which compromises credibility amongst peers. Additional disincentive derives from the academic culture in which principal investigator status on grants and/or senior authorship on papers undermine rewards for collaborators who are essential but do not have either of these leadership roles and therefore do not receive recognition for their contributions. Institutions may deny them promotion as a result. 6,7 Translational studies tend not to be adequately represented within the leadership of academic institutions and are low down in the hierarchy.

Like Pober et al., and the contributors to this book, we have little difficulty identifying opportunities to combine basic and clinical research in our own field (see next section). However, translational research is expensive and more complicated to undertake. Funding of clinical and scientific components tend to come from separate sources. Hypothesis driven research generally tends to be favoured over applied research by funding bodies but is much more difficult to carry out on human subjects as opposed to laboratory models in which the primary criterion is scientific rigour. Academic medical centres appear to have little influence to challenge the prevailing status quo and in support of translational research. Research programmes also need support staff and other resources. For example, writing, administrative, financial and technical skills are needed to run the grants and physically do the projects. Such resources usually come through departments, structures that do not lend themselves to the support of interdepartmental collaborations, which therefore become far more difficult to sustain. Also within an academic medical centre, there is often physical separation between basic scientists and clinicians as they are split into departments within their own disciplines as opposed to multi-disciplinary groups, diminishing communication and therefore understanding and cooperation. Further difficulties arise because clinicians and 
basic scientists have difficulty in recognising the relative merits of each other's work. Institution and institutionalised bureaucracy can also sap energy and unintentionally reduce the ability of committed individuals to deliver high quality research.

Together with all these factors, complying with the deluge of new regulations to protect patients and their rights (essential though they are) add to the researchers' work load and act as further disincentives to research involving humans. There may also be conflict of interests in which the needs of patients are weighed against financial gain. Ethics committees are essential in regulating these aspects but have a fine line in which to balance the relative merits. They champion and safeguard the patient and are a vital component of the system but the translational researcher can be left in a rather solitary position as the only advocate for the research.

\section{SOME CHALLENGES OF PCa}

We believe there are three major challenges where translational research could make a major impact in the management of patients with PCa and those of us who seek to improve their lot. Our examples include:

(1) Identify and distinguish between sub-groups of patients with organ confined $\mathrm{PCa}$ that is (a) clinically significant, genuinely localised, likely to do harm within the patients life time and therefore have the potential to be eradicated with local radical therapy compared to (b) clinically significant and potentially life threatening PCa that are apparently organ confined but where occult metastases have developed and in whom local radical therapy would not impact eventual outcome compared to (c) clinically insignificant, not life threatening $\mathrm{PCa}$ and where radical therapy is unnecessary but if offered, outcome is not impacted and harm could be caused. This challenge will become increasingly important as screening programmes identify potentially large numbers of patients who would not 
otherwise have presented clinically and who may never develop significant disease.

(2) Develop treatments for extra-prostatic disease i.e. that for which there is currently no curative therapy and

(3) Understand the psychological processes involved in decision making on an individual basis as both clinicians and patients explain and rationalise about the management of the disease.

To achieve these will require improved tumour markers (serum/ urine/tissue), improved imaging, better targeting and advances in technology. Patients and researchers will have to spend more time together. There will also need to be a change in culture because as an increasing evidence base accumulates, entrenched opinion from supporters of views at the extremes of the varied management options will, through clinical governance and peer pressure, be required to alter their clinical practice toward the mainstream.

\section{SOME CONTROVERSIES AND PCa}

Much of the current state of knowledge regarding $\mathrm{PCa}$ is covered in the book and we do not intend to give a mini-overview here. However, we felt it would be of interest to look at some of the aspects of $\mathrm{PCa}$ where uncertainty and sincerely held but often entrenched, dogmatic opinion exists without definitive evidence in support. We want to provoke controversy and perhaps identify some areas where the different stake holders and their vested interests could influence judgement. These include the health care industry (doctors, hospitals, pharmaceutical and technology companies, etc) as well as perhaps most importantly patients and their advocate groups themselves. We are all familiar with those who "know" that they can cure $\mathrm{PCa}$ and that screening will decrease mortality equally as well as those who "know" that no such benefits are possible. Hopefully, it will be possible to encourage impartial objective dissection of the evidence with the ultimate aim being beneficial to the patients and their families. 
It may be helpful to review other cancers to examine how changes in perception of disease have impacted mortality and morbidity. PCa remains a relatively new area of research by contrast to for example breast cancer, which has been occupying an increasingly important place in the political agenda for the past 30 years or so. It is astonishing to think that in the late 1960's, there was very little research targeted at women's health. ${ }^{8}$ The surgeons of the early part of the last century believed that the reason for failure of local treatment for breast cancer (i.e. mastectomy) was that it was insufficiently radical. So first they advocated the removal of pectoralis major, then pectoralis minor with axillary lymph node clearance, to be subsequently followed by the internal mammary lymph node chain, requiring thoracotomy. The unfortunate women who underwent this mutilating surgery suffered severe sequelae from treatment but with no identifiable cancer specific survival advantage. The pendulum has since swung the other way and it is now recognised that breast cancer should be considered a systemic disease at diagnosis and managed accordingly. Minimally mutilating surgery to minimise the risk of local recurrence is the current treatment of choice along with adjuvant cytotoxic chemotherapy and/ or hormone therapy to try and impact systemic disease. These recent changes in management have resulted in improvements from an average baseline 10-year mortality of $40 \%$ in 1985 into an increased absolute survival benefit of $10 \%$ by $2000 .^{9}$ These data suggest modern treatment ensures that for every 100 women treated, 10 more are alive today than may have been 15 years ago.

By contrast, men's health has only recently begun to climb up the political agenda and $\mathrm{PCa}$ become one of the key targeted diseases. It is sobering to consider that the first radical prostatectomy was performed in the early 1900's and it is only now, almost 100 years later, that any meaningful trials are reporting its impact in comparison to other radical therapies or watchful waiting. How could this delay have happened? 
Most recently, Holmberg et al. ${ }^{10}$ in a randomised trial of 695 men followed for a median of $>6$ years compared radical prostatectomy and watchful waiting. They reported a significant decrease in disease specific mortality but no significant difference between the two arms in terms of overall survival. The trial began before the onset of PSA screening and was limited to patients with well or moderately differentiated disease only. However, it could be interpreted from these early data that patients may be damned whether they have surgery or not! Most trials should report in the next decade during which period it is to be hoped that management guidelines become clarified. Aggressive local therapy of PCa is becoming more frequently practised and it will be interesting to look back in say 20 years time at the results of current trials (when hopefully the data are mature and the questions will be answered), at its impact on disease outcome. With the current ease of access to massive amounts of information, it has become increasingly difficult to randomise patients. Imaginative strategies such as the ProtecT study $^{11}$ may assist in improving recruitment and enable faster outcome data as patients themselves elect to undergo one or other treatment.

There are other possibly important parallels between breast and $\mathrm{PCa}$, none more so than in the area of screening.

\section{DEVELOPMENTS AND CONTROVERSIES IN SCREENING: WHO IS CONTROLLING THE AGENDA?}

\section{Some Screening Issues}

The kind of emotive responses engendered by potentially lifethreatening illnesses and cancer in particular were dramatically illustrated by a series of commentaries, which appeared recently in the San Francisco Chronicle. ${ }^{12}$ Late in 2001, an article was published about the manager of San Francisco's baseball team. A diagnosis of $\mathrm{PCa}$ had followed "routine" PSA testing and resulted 
in radical, potentially curative, surgery. His doctors were quoted as saying "this was the surest way to prevent return of the disease". The editor and deputy editor of the Western Journal of Medicine (Yamey \& Wilkes) decided to respond by writing what they considered to be a "balancing" article in January 2002 for the newspaper. ${ }^{13}$ The resulting outpouring of vitriol from advocates of PSA screening from all walks of life in response suggested at best a lack of objectivity in interpreting data and at worst a deliberate intention to prevent debate, silence critics and use emotional arguments to push forward without scientific enquiry a screening agenda that even the most fervent advocates of screening will usually agree may also have the potential to do harm. Yamey \& Wilkes also raised the spectre of the influence of vested interests that encourage (or discourage) screening and potentially bias consumers and purchasers.

Advocates of PSA screening bombarded their email in-boxes with accusations, abuse and threats, in which they were compared to Mengele and accused of having the future deaths of hundreds of thousands of men on their hands. Some wrote wishing that they themselves would get PCa. Others argued that only urologists were qualified to talk about PSA testing. Letters flooded into the office of the Chancellor of the University of California and the Dean of the University of California Davis Medical School urging that they be disciplined or sacked. Dick Warder, founder of Prostate Cancer Awareness (www.pca.awareness.net) was sufficiently disturbed to write to the BMJ of his personal experience. ${ }^{14}$ This was of a (so far) successful outcome to radical intervention following screening for his own PCa which was compared to that of an acquaintance with a raised PSA who died of $\mathrm{PCa}$ after declining therapy when asymptomatic. These anecdotal episodes were used as informed arguments in favour of screening along with an unreferenced quote that PCa mortality has declined $18 \%$ from 1993 to 1998 and led to his belief that "screening works" and that he represents "survivors who object to distortion of evidence". In fact, SEER data suggest 
that the five year PCa mortality is slightly higher now than in the 1950 's, despite that five year survival has increased from $40 \%$ in the 1950 's to about 95\% currently. Five-year survival is affected by diagnostic practice: the incidence of PCa in the USA has increased about 3-fold over the same period. Data may appear to be improved by artificial artefacts such as for example deaths postponed (leadtime bias) rather than avoided, a regular criticism of screening. ${ }^{15,16}$ To counter balance Ward's advocacy, unfortunately also with anecdote, Lewis was reported as stating that his father nearly died from the side effects of a prostate biopsy that followed a PSA test (the biopsy result was negative). ${ }^{17}$

At least with $\mathrm{PCa}$ there is an opportunity to test the hypothesis that screening may impact on mortality at an earlier stage of development and many very large studies are underway. Such controversies as above also attach to the breast cancer screening project. However this is now so ingrained in western psychology, with substantial investment of US\$3-4 billion annually and 30 million US women having mammography every year, that randomised trials appear to be almost impossible to conduct. ${ }^{18}$ Charatan quotes an article in the New York Times (NYT) at the end of January $2000^{19}$ describing a committee of cancer experts, the Physician Data Query screening and prevention editorial board (PDQ Board), which had found insufficient evidence to demonstrate that screening mammograms prevented breast cancer death. ${ }^{20}$ An NYT editorial pointed out that a great deal of money was at stake on this issue and predicted considerable difficulty in achieving an independent review of benefits of screening mammography: "a mammography has been so strongly endorsed by the cancer establishment and become such a significant source of revenue... for many hospitals and doctors, that it may be difficult to excise without overwhelming evidence that it is dangerous". A recent Cochrane review concluded that "currently available evidence does not show a survival benefit of mass screening for breast cancer (and the evidence is inconclusive for breast cancer mortality)" ${ }^{21}$ Balancing articles appeared later, 
including those from the National Cancer Institute (which uses the PDQ Board to provide information for its on-line database) and said that women should continue to attend for mammograms. It is very disappointing that it was left to the broad sheet press to conclude the debate (by way of an editorial saying "a serious and open assessment of the data is crucial"), rather than clinicians and scientists.

Wells believed that the debate over screening for breast cancer amongst women in their forties had assumed an importance out of proportion to its potential impact on public health and illustrated some of the many obstacles to putting research findings into clinical practice. She suggested that in the absence of an unequivocal answer, and when professionals responsible for promoting the best interests of patients disengage, involvement of patients interest groups and politicians results in loss of objectivity. ${ }^{22}$

Entrenched arguments in favour of or against screening somehow need to be translated, using solid reliable evidence, into practical advice that is understandable and useable in every day practice by clinicians and patients. It should not be left to the lay press to impress this kind of value judgement on patients and the medical establishment, nor should it be necessary for them to advocate for the need for measured and objective criteria, especially when having to explain difficult and controversial diagnostic and therapeutic options and where the natural history of the disease is still so inadequately understood. Clinicians and scientists need to demonstrate objectively where possible. Where evidence is lacking, they should present current knowledge along with its benefits and limitations.

de Lemos has suggested ${ }^{23}$ that the first step to resolving this controversy should be to ensure that all interested parties agree and understand the questions that need to be addressed. He implied that because of the manner in which the debate had evolved between stakeholders, differences appeared to be irreconcilable because they each had very different numbers in mind needed to 
test. In his view, this should start with identification of how many men need to be tested to identify one PCa or prevent one death. Using contemporary references, he calculated that PSA screening could prevent one death related to $\mathrm{PCa}$ per 100 men tested, accepting that there were potential errors in his calculations.

All the major bodies with an interest in $\mathrm{PCa}$, whether in favour or against screening, support that men receive information sufficient to enable them to make a fully informed decision about diagnosis and future therapy. It has been estimated that this may take at least up to 20 minutes, an important but very costly exercise. ${ }^{24}$

In the debate over screening, it remains unknown, which of these arguments may be right or wrong. Irrespective, attempts at silencing and even destroying critics of PSA screening in what appears to be rational debate seem rather sinister as well as shameful. As a result, we felt even more strongly encouraged to seek out answers and evidence, outcomes likely to be delivered faster and with greater accuracy when clinicians and scientists collaborate together and "bridge that gap".

\section{DECISION MAKING}

Bank's asserts that men do care about health issues, but feel unable to talk about their concerns or often fail to seek help until it is too late. ${ }^{25}$ This is confounded by the impact of social class, morbidity and mortality increasing in direct proportion to the level of depravation.

In order to inform patients and enable them to participate in decision making, it is obviously essential that we know what they want to know on an individual basis. We also need to try and understand our own behaviour and how this impacts on the manner in which we present information and influence the decision making process. Thus, although most urologists acknowledge the importance of quality of life in the treatment of $\mathrm{PCa}$, surgical training demands cure and it can be difficult to reconcile these sometimes conflicting demands with surgical training. ${ }^{26}$ Most patients with PCa appear 
to want to share decision making with the doctor ${ }^{27}$ and to consult with their partner. ${ }^{28}$ There are decision making tools available. ${ }^{29}$ It seems important to understand these points so that whilst for examples oncologists recognise the importance of communication skills and receive training to improve their ability to detect and treat psychological distress, ${ }^{30}$ urologists may not be so aware that there is a problem and training has been lacking. As is increasingly becoming apparent, gender also influences how patients perceive and process information and that psychosocial differences affect coping style, psychological distress and patients partners. ${ }^{31}$ It is therefore not appropriate to extrapolate for example the considerable data currently available on these aspects regarding women and breast cancer and apply it automatically to men with for example PCa. It is important that patients be fully involved in the decision making process. Shared decision making has been recognised as more effective, relevant and appropriate than the traditional patient directed model. ${ }^{32,33}$ Fallowfield $^{34}$ argues that a desire for information does not equate to a desire to participate in decision making. We need ways to identify which patients require what information from us as well as training to deliver these objectives.

\section{INFLUENCE OF HEALTH CARE POLICIES ON PROVISION OF HEALTH CARE}

We have already mentioned the impact of economics and politics on screening issues. In addition, policies on health care financing have also been shown to influence clinical management in many conditions. For example, one study suggests that the absence of insurance for tubal ligation resulted in caesarean section being used as an opportunity for clandestine tubal ligation. ${ }^{35}$ Another suggests that the business ethos in health care in Chile encouraged "pragmatism" amongst doctors who do not have a moral objection to non-medical caesarean section. ${ }^{36}$ Reports of any factors other than patient well being that influence clinicians in managing care may 
compromise our moral high ground as individuals trusted with the guardianship of the care for our patients. The US Institute of Medicine, National Academy of Sciences has stated that where hospitals and doctors improve quality of care for patients, they often lose money. For example, appropriate use of peri-operative antibiotics reduces complications and improves outcomes but decreases income for the hospital. New strategies for incentivising health care systems could have real impact in redefining management. ${ }^{37}$ As clinicians and scientists, we need to demonstrate, and be shown to have demonstrated the highest standards of objectivity without bias if we are to maintain the high level of respect and faith afforded to us by our patients.

\section{CONCLUDING COMMENTS}

This book has come to fruition over four years and a personal input of over 2500 hours. It has proved a huge task, but we have learnt an enormous amount and believe it was worth it. In this chapter, we have skimmed through just a few of the areas we believe are important from a general as well as specific PCa related perspective. We hope the wealth of information in this book will be easy to assimilate, stimulate debate and help contribute to bridging the gap between scientists and clinicians.

We hope the book will be successful and then to apply the lessons learnt to improve further editions in the future. We acknowledge and thank all the contributors for their hard work.

\section{REFERENCES}

1. Marwick, C. (2001). Scientists recall progress and promise of translational research. JNCI, 93: 13-15.

2. Pober, J.S., Neuhauser, C.S. and Pober, J.M. (2001). Obstacles facing translational research in academic medical centres. FASEB, 15: 2303-2313. 
3. Rustgi, A.K. (1999). Translational research: What is it? Gastroenterology, 116: 1285.

4. Kassirer, J.P. (1994). Academic medical centres under siege. NEJM, 331: $1370-1371$.

5. Rosenberg, L.E. (1999). The Physician-Scientist: An essential- and fragile-link in the medical research chain. $J$ Clin Invest, 103: 16211626.

6. Dauphinee, D. and Martin, J.B. (2000). Breaking down the walls: Thoughts on the scholarship of integration. Acad Med, 75: 881-886.

7. Freireich, E.J. (1997). Who took the clinical out of clinical research? Mouse versus man. Clin Cancer Res, 3: 2711-2722.

8. Meryn, S. \& Jaded, A.R. (2001). The future of men and their health. BMJ, 323: 1013-1014.

9. Brown, P. (2000). UK death rates from breast cancer fall by a third. BMJ, 321: 849.

10. Holmberg, L. et al. (2002), A randomised trial comparing radical prostatectomy with watchful waiting in early prostate cancer. NEJM, 347: 781-789.

11. Donovan, J. et al. (2002). Improving design and conduct of randomised trials by embedding them in qualitative research: Protec'T study. BMJ, 325: $725-726$.

12. www.sfgate.com/cgi-bin/article.cgi?file=/chronicle/archive/2002/01/18/ ED135201.DTL

13. Yamey, G. and Wilkes, M. (2002). The PSA storm. BMJ, 324: 431.

14. Ward, R. (2002). Storm over screening for prostate specific antigen. Innuendo in article is insulting. BMJ, 324: 1392.

15. SEER, Incidence and Mortality Trends, 1950-1997. http://seer.cancer. gov/publications/csr1973-1997/overview/13.pdf

16. http://seer.cancer.gov/publications/csr1973-1997/prostate.pdf

17. Tonks, A. (2002). Storm over screening for prostate specific antigen. A summary of responses. BMJ, 324: 1392-1394.

18. Charatan, F. (2002). The great American mammography debate (personal view). BMJ, 324: 432.

19. www.nytimes.com/2002/02/11/health/11MAMM.html?pagewanted=1 
20. www.nci.nih.gov/cancer_information/doc_pdq.aspx?version=provider@ viewid=b906d0d0-63ac-4d55-ac29-2ae992440adf\#6

21. www.cochrane.org/cochrane.revabstr/ab001877.htm

22. Wells, J. (1998). Mammography and the politics of randomised controlled trials. BMJ, 317: 1224-1230.

23. De Lemos, M.L. (2002). Storm over screening for prostate specific antigen. Number needed to test needs to be known. BMJ, 324: 1393.

24. Donovan, J.L., Frankel, S.J., Neal, D.E., Hamdy, F.C. (2001) Screening for Prostate Cancer in the UK. BMJ, 323: 763-764.

25. Banks, I. (2001). No man's land: Men, illness and the NHS. BMJ, 323: 1058-1060.

26. Steiner, M.S. (2000) Quality of life after prostatectomy. J Urol, 163: 870-871.

27. Wong, F. et al. (2000). Men with prostate cancer: Influence of psychological factors on informational needs and decision making. $J$ Psychosom Res, 49: 13-19.

28. Boehme, U., Clark, J.A. (2001). Married couples' perspectives on prostate cancer diagnosis and treatment decision-making. PsychoOncology, 10: 147-155.

29. DePalma, A. (2000). Prostate cancer shared decision: A CD-Rom educational and decision-assisting tool for men with prostate cancer. Semin Urol Oncol, 18: 178-181.

30. Maguire, P., Faulkner, A., Booth, K., Elliott, C., Hillier, V. (1996). Helping cancer patients disclose their concerns. Eur J Cancer, 32A: 78-81.

31. Kiss, A., Meryn, S. (2001). Effect of sex and gender on psychosocial aspects of prostate and breast cancer. BMJ, 323: 1055-1058.

32. Elwyn, G.J., Edwards, A., Kinnersley, P. (1999). Shared decision making in primary care: The neglected second half of the consultation. $\mathrm{Br}$ J Gen Pract, 49: 477-482.

33. Coulter, A. (1997). Partnerships with patients: The pros \& cons of shared clinical decision-making. J Health Serv Res Policy, 2: 112-121.

34. Fallowfield, L. (2001). Participation of patients in decisions about treatment for cancer. BMJ, 323: 1144. 
35. Faundes, A., Cecatti, J.G. (1993). Which policy for caesarean section in Brazil? An analysis of trends and consequences. Health Policy and Planning, 8: 33-42.

36. Murray, S.F. (2000). Relation between private health insurance and high rates of caesarean section in Chile: Qualitative and quantitative study. BMJ, 321: 1501-1505.

37. Crossing the quality chasm: A new health system for the 21st Century. www.nationalacademies.org 